\title{
Value-Creation Dynamics in Platform Ecosystem: A Firm Theory Lens
}

\author{
Dohoon Kim \\ Kyung Hee University \\ dyohaan@khu.ac.kr
}

\begin{abstract}
This paper studies platform ecosystems, a novel business entity built upon a platform coordinating the interactions between suppliers and users, particularly in the IT service sectors. Borrowing words from two core firm theories - resource-based view (RBV) and transaction cost view (TCV)-, we propose that the platform ecosystem works at the boundary between "market and hierarchy." To show this, we develop a conceptual, stylized, dynamic model for the platform ecosystem with typical notions in RBV and TCV: in particular, installed-base, player heterogeneity, and platform's investment as primary ingredients. Our findings from equilibrium analyses and simulations largely confirm that both RVB and TCV are valid for understanding a platform ecosystem. We, however, also identify some contingencies where $R B V$ is limited, and propose a hypothesis that the platform' investment is more crucial for fostering an ecosystem. This implies that the platform starts from the point near market and maneuvers the ecosystem toward a hierarchy, utilizing its investment as a driver.
\end{abstract}

\section{Introduction}

With IT becoming prevalent in our society, the platform business has emerged as a novel solution for production and delivery of various IT services. Facebook, and Google represent the most successful cases of the platform business. Such a new business form, however, usually constructs its own business ecosystem by engaging diverse participants: users, input suppliers, and other types of complementors ([6] [11] [27]). Accordingly, the value creation mechanism undergoes a major transformation. For example, the platforms within their ecosystems do not always show the profit-maximizing behavior ([15] [19]). Further, the value creation mechanism is different from the one in the traditional value chain model ([23]). For example, the way that value is created in the models of twosided market or multi-sided platform ([8] [14] [24]) can be hardly described as a linear value chain. The non-linear interactions among the participants in an industry or an ecosystem can be described with the notion of 'value network ([1] [22]).' However, the platform ecosystems present additional dimensions upon their network structures due to the active role of the platforms ([9] [11]), thereby enhancing non-linear value creation. We observe this nonlinearity in a platform ecosystem, and develop a stylized model from the perspectives of firm theory for deep understanding of its organizational nature.

This study proposes a novel conceptual model that analyzes the value creation mechanism on the basis of interactions across the platform's participants. For acquiring a new perspective on platform ecosystems, we first consider the relationship between market and hierarchy ([29]) in the firm theory. In particular, the value creation mechanism in the platform ecosystems is studied on the basis of two representative perspectives on firms: that is, resource-based view (RBV) and transaction cost view (TCV). According to $\mathrm{RBV}$, the accumulated participants turn into the platform's idiosyncratic assets ([25]). And TCV requires the platform's active responses to reduce the transaction costs across the participants ([7] [11]).

Our stylized model presents an abstraction of the service operations organized by a platform through the lens of both perspectives. We also focus on the leadership of the platform to create a virtuous circle of creating value and sustaining the ecosystem. In particular, we aim to derive answers to the following questions from our model and analysis:

- Will the size of active players (users and suppliers participating in a platform ecosystem) reinforce the platform's leadership in an ecosystem?

- Will the platform's investment to reduce the transaction costs borne by the participants encourage the prosperity of the entire ecosystem?

- What are the contingencies and the drivers that affect the growth path of the platform ecosystem? What implications do these factors have for the platform's role: e.g., market vs. hierarchy?

\section{Market, organization, and platform ecosystem}

\subsection{Understanding of platform ecosystems}

The early development of the value chain models 
helped establish the fundamental framework for linear production systems in the era of mass production ([23]). Here, value is created through the activities of a chain of players, who are arranged in one direction along a straight line starting from the suppliers and ending at the customers. With great recognition of the importance of efficient supply chain management, this model is most suitable for sequential productions with value added step-by-step along the chain.

However, recent phenomena, particularly observed in the IT service sectors, present a new aspect of value creation. This calls for a novel theory to integrate the firm theories in a new style. As is evident from the success stories of global giant platforms such as Apple, Facebook, Google, etc., the current wave characterized by keywords like "social" and "mobile" makes the service lifecycle short and require agility in the value creation mechanism. To cope with these challenges, flexible partnerships are necessary, and a player acting as a pivot is required in many situations. Platforms are now playing this role. They organize resources from diverse partners (e.g., complementors, input suppliers, and distributors) and coordinate their varied interests. As a result, it is no longer remarkable characteristics or technology owned by a single company but a holistic complex body comprising multiple inputs from various participants that determines competitive advantage. Accordingly, the business paradigm has shifted, at least in the IT service sectors, from a stand-alone business model led by a flagship company to a community-like one built upon a platform ([15]).

This outlook indicates that platforms lie at the center of value creation. However, they also present a new challenge in terms of understanding and analyzing the value creation mechanism based on a platform. Traditional frameworks such as the value chain model fall short of capturing the essential features of platform activities. Indeed, many participants with diverse interests in the platform business interact with each other quite differently from those in the value chain. First, the partners of a platform belong to different business fields or industries and form more than a simple supplier-buyer relationship. The structure of their relationships cannot be properly described as a chain of dyadic relations; rather, it is closer to a network. Therefore, a linear model fails in modeling the mechanism of value creation by a platform.

In order to emphasize this difference, we employ the term "platform ecosystem," which is now frequently encountered in literature ([9] [15] [17] [18] [19] [20]). In our platform ecosystem, the platform establishes the strategic position as a keystone and takes charge of as well as responsibility for creating values throughout the ecosystem. All the core transactions are mediated by the platform that hinders service flows from running along multiple paths. This fundamental feature of a platform ecosystem lies in the role of the platform in connecting different needs that otherwise could not access each other ([14]). Thus, a platform creates a new marketplace which could not exist without it (in this regard, the platform works as if it were a market). Moreover, the value in a platform ecosystem is not added in a step-by-step fashion following a chain of dyadic relations; instead, it reveals itself at the final delivery stage.

However, a platform cannot exclusively possess all the resources. Thus, it needs to be open and share some components of its resources in order to foster its ecosystem. However, a platform does not fully open everything. It may open core resources but maintains its control over those resources ([4] [6] [7] [12] [13]). Owing to this loosely coupled governance, the entire ecosystem is able to achieve a balance between openness and control, and makes the entire ecosystem look as if an organization: e.g., a balance between proprietary and non-proprietary assets ([7] [16]).

The delineation above reveals the underlying reason for the linear value chain model not being applicable to the platform ecosystems. In fact, the unique nature of the platform could be confirmed by its behavior in practice. For example, the basic principle upon which a platform organizes and leads an ecosystem - the organizing principle-does not seem to be based on sheer profit-seeking driver ([8] [10] [11] [15] [19] [20]). Rather, the organizing principle seems to emerge as a product of evolution, in which the participants coordinate and compete with each other, and interact with and adapt to various environmental factors. In the course of platform's growth, its fitness largely depends on the efficiency and agility generated from its relationships with its partners.

\subsection{Firm theories applied to platform ecosystem}

The primary objective of our study is to find the key characteristics of platform ecosystems from the organizational perspective. This viewpoint bears not only theoretical but also practical implications since many legislation and policy issues around the platform ecosystems are closely related with their organizational nature. Thus, it is necessary to incorporate some key notions from the firm theory into our framework. We started with following the traditional development of the firm theory, and finally decided to consider two major streams in this area-TCV (transaction cost view) and RBV (resource-based view) - to develop a stylized model representing the essential features of the platform ecosystems through the lens of firm theory.

First, TCV ([5] [29]) requests a platform's aggressive actions to reduce the transaction costs 
across the participants: e.g., developing efficient interfaces, standardizing inter-operations, providing SDK. Meanwhile, RBV ([2] [3] [21] [26] [28]) asserts that the network woven by the participants in an ecosystem generates new assets. For example, the fact that the connections between the participants should be mediated by a platform empowers the platform and strengthens its keystone position: e.g., leveraging the network externality. Thus, RBV suggests that the new assets generated by the forging the links between the participants establish core competency for the prosperity of the ecosystems (e.g., accumulated participants as a platform's idiosyncratic asset; [25]).

In our stylized model, the platform tries to leverage both transaction efficiency (TCV) and asset specificity (RBV). The goal of our modelling and analyses is to gain an insight into the role of platform leadership toward a value-creation virtuous cycle throughout an ecosystem. Specifically, we are interested in the following aspects of the platform ecosystems:

- Investments on platform infrastructure ('infra' in brief): Infra provided by a platform is a fundamental base of value creation for the sake of the participants in its ecosystem. For this purpose, the platform needs to regularly invest in its infrastructure so that it can maintain and improve transaction efficiency.

- Participatory base: The participants such as complementors not only give a boost to an ecosystem but also contribute as an idiosyncratic asset to the platform, for example, by attracting other potential participants. Because of this feature of the participants, the platform provides many incentives to expand its participatory base (e.g., free subscription, low licensing fee, subsidy, etc.).

- Holistic ecosystem value: The total value created through an ecosystem does not necessarily translate directly into profits of the platform (this makes the value creation mechanism nonlinear, as explained above). However, the platform still undertakes the responsibility for the holistic value. In this regard, the platform can be viewed to exist not for maximizing its profit but for fostering the ecosystem by inducing potential participants to join the system and promoting transactions across the participants.

\section{Service operations in platform ecosystem}

\subsection{Platform ecosystem model}

In our model, the platform ecosystem is composed of three echelons: (potential) suppliers - platform (potential) users. Potential suppliers and users are horizontally differentiated. As for the user group $U$, a user is given an index $u$ according to his/her preference toward service diversity, which is assumed to be determined by the number of suppliers on the other side. Suppliers are similarly differentiated based on the level of cost or inefficiency. Specifically, we define $U \equiv[0, \bar{u}]$ and $S \equiv[0, \bar{S}]$, where users and suppliers are populated over the sets $U$ and $S$, respectively. Potential users and suppliers are horizontally differentiated according to the functions $d(u)$ and $q(s)$, each of which represents the preference strength of user $u$ and the cost (inefficiency) factor of supplier $s$, respectively. Users are populated on $U$ in a descending order of $d(u)$ $\left(d^{\prime}(u)<0\right)$. On the other hand, suppliers are arranged in an ascending order of $q(s)\left(q^{\prime}(s)>0\right)$.

The payoff functions of a user $u \in \mathrm{U}$ and a supplier $s \in \mathrm{S}$ are defined as follows $\left(\zeta_{t} \neq 0\right.$ assumed). Hereafter, the subscript ' $t$ ' represents the corresponding time period. (For the sake of enhanced readability, however, we sometimes drop ' $t$ ' when its omission does not seem to cause any confusion.)

$\begin{array}{ll}\mu(u)=d(u) \zeta_{t} s_{t}-r_{t} & \text { Eq. } 1 \\ \pi(s)=r_{t} u_{t}-s_{t} q(s) / \zeta_{t} & \text { Eq. } 2\end{array}$

Eq. (1) indicates that user's payoff is determined by the strength of preference toward the diversity and the service fee $r_{t}$ that he/she pays for the services delivered through the platform. Furthermore, the benefit is proportional to the level of efforts $\left(\zeta_{t}\right)$ that the platform puts on for maintaining and improving transaction quality. Thus, this investment is assumed to strengthen the indirect network externality.

Suppose that each supplier provides one unit of input or contribution (either product or service). Then, with given price $r_{t}$ for their services, the payoff of supplier $s(\in \mathrm{S})$ is set by Eq. (2). The suppliers' benefits are also affected by the positive indirect network externality from the user side. However, there is a negative network externality in the supplier side, which indicates the competition costs imposed upon the active suppliers. Hereafter, the former (positive) externality will be simply called the "network effect" and the latter the "congestion effect." Also note that the investment of the platform helps to improve suppliers' transactions, thereby reducing the actual operating costs and alleviating the congestion effect.

Now, our platform seeks its profit not by leveraging price structure (as typical platforms in two-sided market models do) but by managing the installedbases, $u_{t}$ and $s_{t}$. This assumption fits well our viewpoint on the platform described in Section 2 (also refer to the role of platforms explained there). Further, we also assume a myopic platform since the behaviors 
of most platforms in practice do not seem to be consistent with those of perfectly forward-looking players. Therefore, at the point of decision (refer to Fig. 1), the platform's concerns reduce to one step change (i.e., net increase or decrease during the corresponding period) of participants.

And many platforms in practice are trying to maintain (and hopefully increase) their installed-bases and put much efforts (e.g., marketing, subsidy, and other investments) on competitive segments. Thus, the actual behavior of platforms could be described as seeking for an optimal balance between the net increase of the bases and the amount of efforts invested. Accordingly, the platform is assumed to focus only on the gains from the newcomers in both sides $\left(\Delta u_{t}\right.$ and $\Delta s_{t}$ ) in $t$. Now, the platform faces the following decision problem in each period of time $t$.

$$
\begin{aligned}
& \text { maximize } \zeta_{t} \quad \Pi_{t}=a \cdot \Delta u_{t}+b \cdot \Delta s_{t}-c \cdot \zeta_{t} \\
& \text { s.t. } \quad 0 \leq \zeta_{t} \leq B_{t}
\end{aligned}
$$

The payoff is comprised of the net earning increments from both sides and the investment expenditure. Since $\Delta u_{t}$ and/or $\Delta s_{t}$ may be negative (e.g., when there are more players - either users or suppliers-leaving the ecosystem than those joining it in $t$ ), Eq. (3) actually could mean a loss minimization in some periods. We will derive the net changes later (refer to Eq. (6) and (7) and the notion of 'critical player' introduced just before these equations).

The platform also needs to deliberate on the investment. Indeed, many studies on platform businesses assert that one of practical challenges they are facing (in particular, at the early stage of launching and stabilizing the platform) is to commit a big investment in order to keep running the projects for redesigning the value creation mechanism and enhancing associated value flows. We will also show that both $\Delta u_{t}$ and $\Delta s_{t}$ are the functions of $\zeta_{t}$ in our dynamics introduced and analyzed in the next section. Accordingly, the optimization problem above is not trivial as shown in Proposition 5 in Section 4.

However, the amount of investment is bounded by the available budget at the current time, which is also dynamically determined by the ultimate sources of the gains from its value creation. The value created in the platform ecosystem is fed back to the system in the next period. The ecosystem grows based on this feedback mechanism, which specifies the dynamics of our stylized model. That is, a large portion of this feedback flows throughout the entire ecosystem and eventually affects the platform's capability such as financial budget. Thus, the budget capacity in the next period $B_{t+1}$ will be adjusted due to the change in the number of active players, the idiosyncratic asset of the platform according to RBV. Specifically, investment capability of the platform changes according to not only $\Delta u_{t}$ but also $\Delta s_{t}$. The rate of change in $B_{t}$ is assumed to be proportional to a convex combination of the rates of changes in $u_{t}$ and $s_{t}$. With $\gamma$ for this parameter, Eq. (4) represents a general representation of the budget dynamics from $B_{t}$ to $B_{t+1}$.

$B_{t+1}=B_{t}\left[1+\gamma \frac{\Delta u_{t}}{u_{\mathrm{t}}}+(1-\gamma) \frac{\Delta s_{t}}{s_{t}}\right]$

Eq. 4

, where $0 \leq \gamma \leq 1$.

Note that the payoffs are determined in a nonlinear way and the ecosystem creates the value differently from what traditional value chains do. For example, the platform should take care of not only its profit but also the holistic ecosystem. Therefore, the payoff function of the platform becomes quite different from the additive form employed in linear value chain models.

Another major distinction comes from the interrelationship between users and suppliers. The relationships among the three players form a triad. That is, the connection between a user and a supplier is established via the platform while the actual service flows (business transactions) occur between the user and the supplier. This feature is reflected in the way of determining $r_{t}$, the service fee between users and suppliers at time period $t$. Unlike traditional value chains where a keystone player with negotiation power usually sets and controls $r_{t}$, the major revenue streams for the platform come from the size of participants in its ecosystem. Since our model assumes a plentiful of users and suppliers, the price $r_{t}$ will be determined by the typical principle of supply and demand; that is, both users and suppliers behave as a price taker. Accordingly, we incorporate an adaptive dynamics frequently employed in the studies with the supplydemand interactions above. Thus, the price change $\Delta r_{t}$ is supposed to follow the equation below:

$\Delta r_{t} \equiv r_{t+1}-r_{t}=\delta_{u} \frac{\Delta u_{t}}{u_{t}}-\delta_{s} \frac{\Delta s_{t}}{s_{t}} \quad$ Eq. 5 , where $\delta_{u}$ and $\delta_{s}$ are positive constant weights $(<1)$.

Lastly, the number of users [suppliers] in the next time period is determined by a convex combination of the current size $u_{t}\left[s_{t}\right]$ and the deviation from the 'critical' size $u_{t}^{c}\left[s_{t}^{c}\right]$, where $u_{t}^{c}$ is defined as the corresponding index of the user [supplier] who is indifferent between joining and leaving the system: i.e., $\mu\left(u_{t}^{c}\right)=0\left[\pi\left(s_{t}^{c}\right)=0\right]$. Note first that the critical user [supplier] is well defined since users [suppliers] are arranged and indexed in descending [ascending] order of $d(u)[q(s)]$ over $\mathrm{U}[\mathrm{S}]$. Thus, the critical user [supplier] may well represent the expected size of active users [suppliers]. 
$\Delta u_{t} \equiv u_{t+1}-u_{t}=\alpha\left(u_{t}^{c}-u_{t}\right)$

Eq. 6

$\Delta s_{t} \equiv s_{t+1}-s_{t}=\beta\left(s_{t}^{c}-s_{t}\right)$

Eq. 7

, where $0 \leq \alpha, \beta \leq 1$.

Here, $\alpha$ and $\beta$ are adjustment parameters (as $\gamma$ in Eq. 4) in the respective adaptive processes. For example, $\alpha=1$, users instantaneously respond to the critical player $\left(u_{t}^{c}\right)$, and rapidly adjust themselves toward the point. With $\alpha[\beta]=0$, meanwhile, users [suppliers] do not switch and $u_{t}\left[s_{t}\right]$ remains fixed.

For example, with given $\zeta_{t}$ set by the platform and other parameters at the beginning of $t$, (potential) users decide whether to join the ecosystem or not. They regard the number of suppliers $s_{t}$ as given at the moment (actually updated at the end of the previous period; i.e., $s_{t}=s_{t-1}$ ). In the course of (potential) users' movement-staying, joining, and leaving —, the critical user $u_{t}^{c}$ is determined. It will be quite common in practice, however, that there is a gap between the expected number of users $\left(u_{t}^{c}\right)$ and the size of actual users at the beginning $\left(u_{t}\right)$, which results in $u_{t}^{c} \neq u_{t}$ in general. The same adjustment procedure applies to the supplier side (with $u_{t}, s_{t}$, and $s_{t}^{c}\left(\neq s_{t}\right.$ in usual)). Because of these mismatches, some users and/or suppliers may experience negative payoffs for a while. Upon this gap, the size of active users [suppliers] in the next period, $u_{t+1}\left[s_{t+1}\right]$ is adjusted based on Eq. (6) [Eq. (7)]. Fig. 1 summaries the event sequence in our stylized adjustment process.

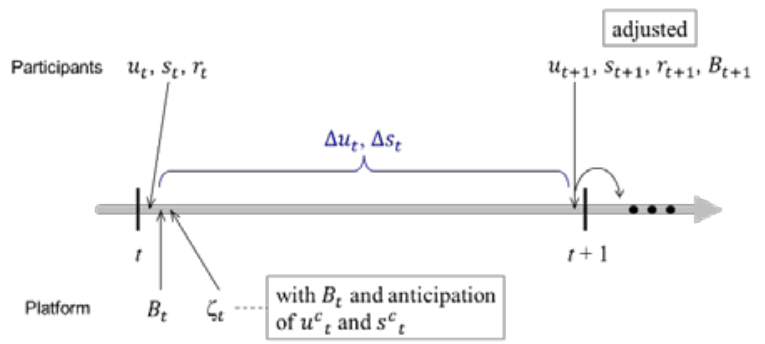

Figure 1. Timeline

Platform's investment decision is assumed to be accomplished at the beginning of each time period. This action triggers the adjustment process and eventually leads to a new state for the next period $t+1$.

\subsection{Steady state equilibrium analysis}

We now analyze the steady state equilibriums in the platform ecosystem with respect to the payoff functions (Eq. (1) and (2)). First, we define the possible types of equilibrium as follows:

- Interior equilibrium (eq.): $\left(u^{*}, s^{*}\right)$, where $u^{*}$ and $s^{*}$ belong to $(0, \bar{u})$ and $(0, \bar{s})$, respectively,

- Boundary eq. at the full saturation: $(\bar{u}, \bar{s})$,

- Boundary eq.'s of partial saturation: $\left(\bar{u}, s^{c}\right)$ or $\left(u^{c}, \bar{s}\right)$, where $u^{c}<\bar{u}$ and $s^{c}<\bar{s}$,

- Boundary eq. at the complete collapse: $(0,0)$.

We do not consider the equilibria where only one side (user or supplier) vanishes in the ecosystem. That is, we accept neither $\left(0, s^{c}\right)$ nor $\left(u^{c}, 0\right)$ as an admissible state. This exclusion is quite reasonable since they look absurd; for instance, $\left(0, s^{c}\right)$ means a certain number of suppliers without a single user.

The following Proposition says that the boundary equilibrium at the complete collapse $(0,0)$ could be a Nash equilibrium. (Since we deal with a steady state, we omit the subscript ' $t$ ' in the following propositions and explanations for readability.)

Proposition 1 (Complete Collapse Nash Equilibrium) The boundary equilibrium where both sides of the ecosystem collapse is a Nash equilibrium if $r>0$.

\section{Proof (Omitted; to be provided upon request)}

One needs to be careful when interpreting Proposition 1. First of all, the condition $(r>0)$ is not a sufficient one. That is, there could be many possibilities where other types of state become equilibrium with $r>0$. Thus, we should not conclude that the collapse is more likely to occur in the platform ecosystem. On the contrary, it will be shown that other types of the boundary equilibriums are rather more prevalent not only in practice but also in theory. Numerical simulations in the next section also demonstrate that the collapse does not seem prevalent in the platform ecosystem.

Now, it is necessary to examine the other states for a Nash equilibrium. For this purpose, we first need to explicitly express the critical players in terms of the corresponding parameters and the decision variable of the platform. For example, the critical user in our model locates at $u_{t}^{c}=\mu^{-1}(0)$. First, we assume that all the players are homogenous: i.e., $d(u)=d$ for all $u \in \mathrm{U}$ and $q(s)=q$ for all $s \in \mathrm{S}$, where both $d$ and $q$ are positive constants. After analyzing the homogenous case, we deal with the heterogeneous players in one side with linear differentiation (e.g., $q(s)=q s$, where $q$ is a positive constant). More general cases where both users and suppliers are heterogeneous will be dealt with in the experiments.

The following Propositions present possible steady state equilibriums together with respective conditions under the corresponding assumptions on homogeneity of players. Since the complete collapse state also constitutes a Nash equilibrium in the following cases (note that there is no restriction on the players' variety in Proposition 1), we omit this boundary equilibrium in our statement of the following Propositions. 


\section{Proposition 2 (Homogenous Players)}

Suppose that $\zeta$ be given by the platform. In the platform ecosystem with homogeneous players (i.e., $d(u)=d$ and $q(s)=q, d$ and $q$ are constants), we can observe in a steady state that the full saturation state $(\bar{u}, \bar{s})$ becomes a Nash equilibrium when the following inequalities hold:

$$
\frac{r}{d \zeta} \leq \bar{s} \leq \frac{r \zeta}{q} \bar{u} .
$$

Proof (Omitted; to be provided upon request)

The proof of Proposition 2 also implies that there is no interior equilibrium in the case of homogeneous players. Furthermore, the other types of boundary equilibriums except the full saturation state (i.e., partial saturation states) cannot be sustained as a Nash equilibrium. This implies a possible contingency where the installed-bases determine the platform's maximum payoffs, thereby serving as an ultimate source of platform's growth. The platform's investment, however, is also involved in this contingency (refer to the condition in Proposition 2, which holds for large

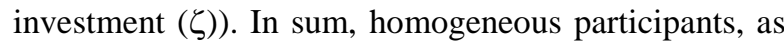
idiosyncratic assets for the platform (RBV), may exert influence on the ecosystem, but this potential can be realized and enhanced through the platform's efforts to improve the relevant transactions (TCV).

\section{Proposition 3 (Heterogeneous Suppliers)}

Suppose that $\zeta$ be determined and given. In the platform ecosystem with homogeneous users $(d(u)=$ $d$ ) and heterogeneous suppliers with $q(s)=q s$ (the second $q$ is a positive constant), we can observe in a steady state that each of the following states becomes a Nash equilibrium when the corresponding conditions are satisfied. Here, $s^{c}=\sqrt{r \zeta \bar{u} / q}$ :

a) $(\bar{u}, \bar{s})$, the boundary equilibrium at the full saturation state with $r /(d \zeta) \leq \bar{s} \leq s^{c}$,

b) $\left(\bar{u}, s^{c}\right)$, the boundary equilibrium at a partial saturation state with $r q /\left(d^{2} \zeta^{3}\right)<\bar{u}$ and $s^{c}<\bar{s}$.

\section{Proof (Omitted; to be provided upon request)}

Similar to the all-homogeneous case, an interior equilibrium does not exist. However, we now have another type of boundary equilibrium with a partial saturation in the supplier side. To put this situation in another way, even with the saturation of the user side, there are some suppliers who would not join the ecosystem due to their inefficiencies in making business transactions with users. Examining $s^{c}$ in the Proposition, we know that increasing investment will maneuver the partial saturation state into the full saturation one (i.e., increasing $s^{c}$ beyond $\bar{s}$ ) since it reduces the transaction costs and makes suppliers' participation more affordable.

\section{Proposition 4 (Heterogeneous Users)}

Suppose that $\zeta$ be determined and given. In the platform ecosystem with homogeneous suppliers $(q(s)=q)$ and heterogeneous users with $d(u)=$ $d(\bar{u}-u)$ (the second $d$ is a positive constant), we can observe [cannot observe] in a steady state that the following candidate states constitutes a Nash equilibrium when the corresponding condition holds:

a) $\left(\bar{u}, s^{c}\right)$, where $s^{c} \in(0, \bar{s}]$, cannot be a boundary equilibrium with $r>0$,

b) $\left(u^{c}, \bar{s}\right)$, where $u^{c}=\bar{u}-r /(d \zeta \bar{s})$, is a boundary equilibrium at the partial saturation state with $\bar{u}>$ $\frac{1}{\zeta}\left(\frac{r}{d \bar{s}}+\frac{q \bar{s}}{r}\right)$.

Proof (Omitted; to be provided upon request)

The analytical results about the steady state equilibriums in the platform ecosystem indicate that the heterogeneity of players has an effect on the structure of the equilibriums. For example, there is a possibility of the full saturation in the case of homogeneous users (Proposition 2 and a) in Proposition 3); whereas the full saturation state cannot be a boundary equilibrium with heterogeneous users due to the following interactions between users and suppliers (when other parameters remain the same). Once the behavior dynamics (Eq. (6) and (7)) leads the state to a point near to $(\bar{u}, \bar{s})$, some users may find out that it is not beneficial to stay in the ecosystem and start to leave it. In the course of this adjustment, the dynamics cannot keep pushing the state toward the corner point $(\bar{u}, \bar{s})$, and it settles down at $\left(u^{c}, \bar{s}\right)$ in the end (see b) in Proposition 4). This asymmetry seems to arise from the difference in the mechanism where the platform's investment $\zeta$ affects the respective players' payoffs.

Facing the situations where at least one side is composed of heterogeneous participants, the platform's leading role through investments seems more crucial to fostering the ecosystem than other initiatives directly aiming at the installed-bases. In other words, with heterogeneous participant configuration (e.g., highly differentiated), transaction efficiency is essential to triggering the ecosystem growth (TCV). Meanwhile, the role of active players as idiosyncratic resource (RBV) is relatively meager; once started, the dynamics draws participants until it reaches a partial saturation.

The platform's decision on the investment scale also plays an important role in determining boundary equilibriums in the contingencies above. For example, the full saturation as an equilibrium with homogeneous 
users requires that the platform should maintain a large amount of investment (refer to the inequalities in Proposition 2 and those in a) of Proposition 3). The boundary equilibrium that occurs along the edge of $s=\bar{s}$ (i.e., saturation of supplier side) with heterogeneous users (refer to b) in Proposition 4) also presumes a sufficient amount of investment from the platform. Also note that $s^{c} \rightarrow \bar{s}$ in Proposition 3 and $u^{c} \rightarrow \bar{u}$ in Proposition 4 as $\zeta$ increases sufficiently. Therefore, we need to explicitly consider the platform's investment decision $(\zeta)$, which will be dealt with in the next section in a more general framework (i.e., heterogeneities both in users and in suppliers).

The analytical results in this section have been derived from a long-term perspective which presumes that the decision of the platform is stabilized. The actual dynamics, however, could alter the static equilibrium especially when the equilibrium causes a behavioral change of the platform and destroys the condition for the corresponding equilibrium. Unfortunately, one could not conduct a rigorous analysis for such possibilities without resorting to some numerical simulations. In the next section, we present a series of experiments and investigate some growth paths that representative scenarios could generate.

\section{Dynamics simulations and discussions}

In this section, the equilibriums in a platform ecosystem are analyzed by explicitly incorporating the platform's dynamics and decisions. We first derive the optimal decision of the platform. Here, it is assumed that the platform's moves are myopic (with its best guess about the change during the period), even though it can produce an optimal outcome in some periods. For the sake of explicit analysis, linear differentiation functions for $d(u)$ and $q(s)$ are assumed as before, that is, $d(u)=d(\bar{u}-u)$ and $q(s)=q s$, where both $d$ and $q$ are positive constants (also see Propositions 3 and 4). Thus, we now consider a more general situation in which both users and suppliers are heterogeneous. Then, on the basis of the decision rule below, we conduct a series of numerical simulations to examine the dynamic performance of the ecosystem.

\section{Proposition 5 (Optimal Decision of Platform)}

Given $u_{t}, s_{t}, r_{t}$ and $B_{t}$ (all positive) at the beginning of period $t$, let's define three constants (over time period $t$ ) $\Phi_{t} \equiv c q s_{t}-b \beta r_{t} u_{t}, \Theta_{t} \equiv$ $\sqrt{a \alpha q r_{t} /\left(d \Phi_{t}\right)}$ (defined only for positive $\Phi_{t}$ ) and $\Psi_{t} \equiv \sqrt{a \alpha r_{t} /\left(c d s_{t}\right)}$. Platform's optimal decision in Eq. (3) on the amount of investment $\zeta_{t}^{*}$ at $t$ follows the rule below:

CASE I : $u_{t}^{c}$ and $s_{t}^{c}$ belong to $(0, \bar{u})$ and $(0, \bar{s})$ resp.
(1) if $\Phi_{t}>0$ and $\Theta_{t}<B_{t}$ then $\zeta_{t}^{*}=\Theta_{t}$,

(2) otherwise, $\zeta_{t}^{*}=B_{t}$.

CASE $\Pi$ : Either $u_{t}^{c}$ or $s_{t}^{c}$ (but not both) is out of its respective range, $(0, \bar{u})$ or $(0, \bar{s})$.

(3) with $u_{t}^{c}<0$ (and $\left.s_{t}^{c} \in(0, \bar{s})\right), \zeta_{t}^{*}=B_{t}$ if $\Phi_{t}>0$ and $\zeta_{t}^{*}=0$ otherwise.

(4) with $s_{t}^{c} \geq \bar{s}$ (and $u_{t}^{c} \in(0, \bar{u})$ ), $\zeta_{t}^{*}=\Psi_{t}$ if $\Psi_{t} \leq$ $B_{t}$ and $\zeta_{t}^{*}=B_{t}$ otherwise.

CASE III: Neither $u_{t}^{c}$ nor $s_{t}^{c}$ belongs to its resp. set. (6) $\zeta_{t}^{*}=0$.

Proof (Omitted; to be provided upon request)

With the optimal responses of the platform summarized in Propositions 5 and the dynamics described by Eq. (4)-(7), a series of simulations are carried out. On the basis of these experiments, we ascertain whether the active players constitute effective resources for the platform (as RBV asserts) and whether the investment to improve the transaction efficiency effectively nurtures the ecosystem (as TCV asserts). We are also interested in some factors that are most influential in leading the ecosystem to a specific state (or scenario). In particular, we try to understand the relevant contingencies and the drivers in terms of either RBV or TCV or both. Along this line, we employ the following performance measures.

- The sizes of active players in period $t: u_{t}$ and $s_{t}$,

- The platform's profit and capital turnover (rate) at $t$ : $\Pi_{t}$ and $\kappa_{t} \equiv \Pi_{t} / \zeta_{t-1}$,

- The average payoffs of active players at $t: \bar{\mu}_{t} \equiv$ $\frac{1}{u_{t}} \int_{0}^{u_{t}} \mu(u) d u$ and $\bar{\pi}_{t} \equiv \frac{1}{s_{t}} \int_{0}^{s_{t}} \pi(s) d s$.

These measures are developed to answer our research questions raised in Introduction. For example, the scales of active players $\left(u_{t}\right.$ and $\left.s_{t}\right)$ are directly linked with our first question that whether the active players could reinforce the platform's leadership in the ecosystem, thereby playing the role of an idiosyncratic asset, as suggested by RBV. Furthermore, the capital turnover $\left(\kappa_{t}\right)$ in the second performance measure is devised to determine the platform's incentive to enhance its infrastructure for improving its transaction efficiency, primarily from the perspective of TCV. The last two measures $\left(\bar{\mu}_{t}\right.$ and $\left.\bar{\pi}_{t}\right)$ monitor the effectiveness of the platform's activity (e.g., investment) in creating a virtuous cycle throughout an ecosystem, thereby sustaining and fostering the whole ecosystem. Evaluating the platform ecosystem from a wide range of angles will enhance our understanding of the platform's role as a keystone player ([15] [18]).

The following figures depict outcomes from an 
example in which the parameters are fixed at certain values (for details, please refer to the description of the baseline experiments given below Fig. 2). We tried tuning the parameters throughout some pilot tests. For example, speed parameters such as $\alpha$ and $\beta$ were tuned to be small; otherwise, the system adjusts itself too fast to properly reveal its dynamic behavior. Despite small $\alpha$ and $\beta$, the platform ecosystem stabilized in all the instances we tried. Thus, this manipulation for slow adaptation is maintained in the following simulation results.

The simulation outcomes are categorized into some typical patterns, which could be justified by extensive trials rich enough to encompass a large number of instances. Fig. 2 demonstrates three most typical patterns of the system behavior.

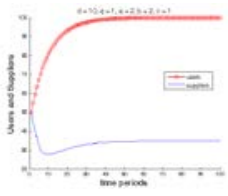

(a) Scenario $A$

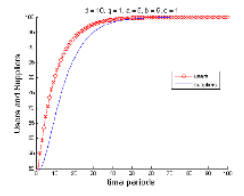

(b) Scenario B

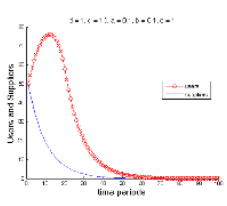

(c) Scenario C
Figure 2. Changes in Users and Suppliers

We set the parameters at the following numerical values: $\alpha=\beta=$ $0.1, \gamma=0.5, \delta_{u}=\delta_{s}=0.5$ and $\bar{u}=\bar{s}=100$. As the initial states, we set $u_{0}=s_{0}=50, r_{0}=2$, and $B_{0}=a b c$. For the first two scenarios, we set $d=10$ and $q=1$ in order to make the comparisons as fair as possible. However, these settings cannot be maintained for generating the all-collapse case; we should, for example, set $d=1$ and $q=10$ to craft Scenario C. Note that the platform's cost is set to be one for all the three scenarios. We run the simulations over 100 time periods (see the horizontal line). These parameters as well as the initial states remain same in the following figures.

Each scenario corresponds to a specific steady-state equilibrium suggested in the previous section. For example, Scenarios B and C represent full saturation and complete collapse, respectively. Scenario A shows a partial saturation instance that resembles the equilibrium $\left(\bar{u}, s^{c}\right)$ in Proposition 3 , though the context assumed there (i.e., homogenous users) is different from that assumed in the simulation (i.e., heterogeneous players). Also note that the small difference between perfectly homogeneous users in Proposition 3 and a little heterogeneity of users in Scenario C produces opposite outcomes: the (possible) full saturation in the former vs. the complete collapse in the latter. In particular, Scenario $\mathrm{C}$ implies a specific contingency where neither active players nor platform's investment works for prosperity of the ecosystem. Strong heterogeneities (particularly, across suppliers) seems like a source of this contingency.

We focus on the first two scenarios: Scenarios A and B. Fig. 3 and 4 display and compare changes in the profits and capital turnover of the platform both in the early phase and steady state; Fig. 3 and 4 respectively depict the outcomes under Scenarios A and B. They show plots for the two performance measures of the platform against the market scale in the early phase as well as near the last phase. In many experiments, we observed that the overall qualitative aspects (e.g., pattern and graph shape) of the system behavior (in terms of the respective measure) remained unchanged.

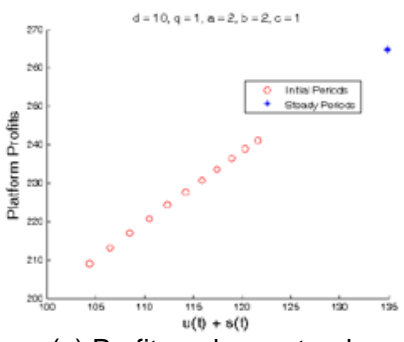

(a) Profit: early vs. steady

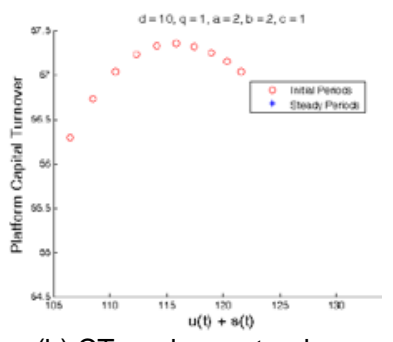

(b) CT: early vs. steady
Figure 3. Changes in Platform's Profits and Capital Turnover (CT) in Scenario A



(a) Profit: early vs. steady

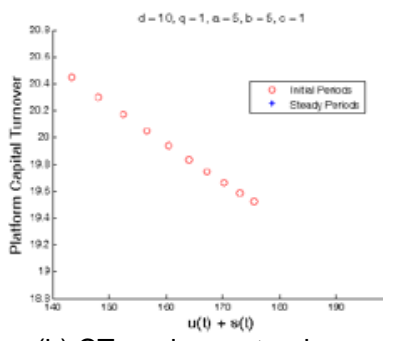

(b) CT: early vs. steady
Figure 4. Changes in Platform's Profits and Capital Turnover (CT) in Scenario B

Both scenarios show that the platform's profit increases as more players join the ecosystem (Fig. 3(a) and 4(a)). Thus, the outcomes seem to support the argument that users and suppliers play the role of idiosyncratic assets, as asserted by RBV ([25]). Considering the capital turnover as the measure of the platform's performance (Fig. 3(b) and 4(b)), however, we see an opposite relation between the ecosystem scale and platform's performance. Thus, we could not uniformly conclude that active users and suppliers constitute the ultimate source of profitability for the platform. We need to further investigate the role of investment at the level of the holistic ecosystem.

The following figures display and compare changes in the average payoffs of active players against the amount of investment in the previous time period (since our event sequence assumes that the players' adjustments are completed at the end of a period while the investment is in effect from the beginning of the period). Both Scenarios A and B result in similar outcomes. For example, the average payoff of active users consistently improves as the investment increases (Fig. 5(a) and 6(a)) thanks to the platform's efforts to 
enhance the service value through investments. On the other hand, the average payoff of active suppliers increases along the scale of investment in the early phase; however, near the steady state, they eventually settle down at a point, which is lower than the ones in the starting periods (Fig. 5(b) and 6(b)). This drop in efficiency gain is attributable to the congestion effect, which becomes stronger as the user side gets saturated (also note that the platform's investment becomes fixed as the ecosystem approaches to a stead state). Thus, the average gain of active suppliers results in an inverted U-shape. This phenomenon seems to be independent of the size of active players since the numbers of active suppliers in the steady state differ between the two scenarios $\left(s_{t} \rightarrow s^{c}\right.$ in Scenario $\mathrm{A}$ and $s_{t} \rightarrow \bar{s}$ (i.e., saturated) in Scenario B). The different behaviors between users and suppliers result from the different payoff structures (see Eq. (1) and (2)), particularly owing to the congestion factor (i.e., the negative network externality) in the suppliers' payoff.

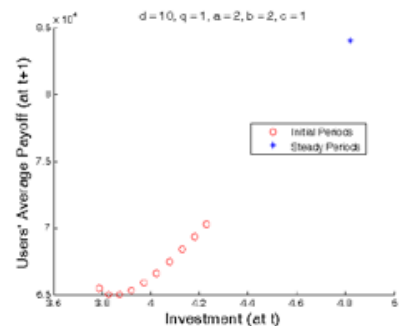

(a) User AP: early vs. steady

Figure 5. Changes in Avg.

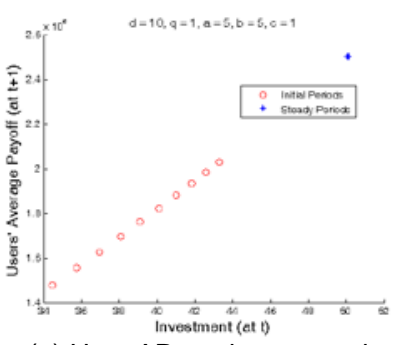

(a) User AP: early vs. steady

Figure 6. Changes in Avg. Payoffs (AP) in Scenario B

However, this contrast between the users and suppliers should not lead to an underestimation of the role of investment in creating value and nurturing the ecosystem. Particularly, the active players - both users and suppliers - in the early phase enjoy positive payoff streams thanks to the platform investment, since it reduces the costs for transactions between two groups, as asserted by TCV. Players' gain or loss may result from sacrifice by or exploitation of the platform in the course of its value creation and propagation.

To examine more closely these profitability links, we demonstrate the platform's payoffs together with those of participants in Fig. 7. Here, we observe coprosperity in both scenarios at least in the early phase, which implies a positive feedback loop of payoff streams for all the participants in an ecosystem. This co-prosperity, however, ends when one side (here, the user side) reaches the saturation state. Thereafter, the platform starts to rein back the investment (not displayed in the figures), and some suppliers suffer from increased transaction costs despite having enjoyed the saturated user side. Furthermore, diminishing average payoff on the supplier side could be aggravated owing to the congestion effect.

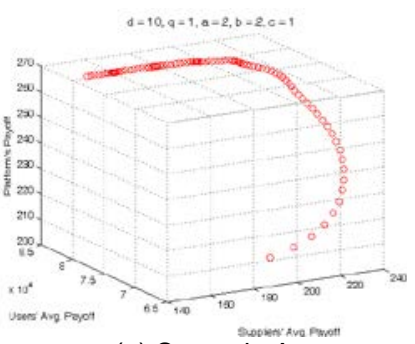

(a) Scenario A



(b) Scenario B
Figure 7. Profitability Links in Platform Ecosystem

Thus far, our experiments show that a platform ecosystem is able to sustainably leverage idiosyncratic assets as well as transaction efficiency, the two key notions that constitute the modern firm theory.

However, users and suppliers not only serve as assets but also consume other platform resources. Accordingly, they cannot be as perfectly idiosyncratic as a proprietary asset that is not available to the participants. Furthermore, the congestion effect on one side (here, the supplier side) is likely to undermine the potential of active participants. This presents a challenge to discover another core asset for a platform ecosystem, which better complies with RBV.

On the other hand, the function of investment for improving the transaction efficiency between the participants seems more critical to ecosystem management. The platform's efforts to reduce transaction costs foster the entire ecosystem and coincide with participants' proliferation (at least in the early phase of its growth in Fig. 7). Further, some contingencies such as heterogeneity in participants dwarf the potential of active players as idiosyncratic assets for the platform. Subsequently, the following hypothesis could be proposed; "with heterogeneous participants, the fundamental rationale of existence of the platform stems from its initiatives more compatible with TCV than RBV." Going one step further, our findings also confirm a general observation on the typical life-cycle behavior of the platform ecosystem and explain this behavioral pattern. That is, a platform starts to create a marketplace and promote its 
ecosystem, and after building up a certain scale of installed-base, it maneuvers the ecosystem toward a hierarchy. And platform's investments work as a main driver that leads and moderates this movement along the spectrum of market vs. hierarchy.

This point provides insight into the platform's role in its ecosystem. For example, the platform needs to conduct a maneuver for the congestion side and suppress surging participants, if necessary, for sustainable growth of its ecosystem. (This point also explains another reason for the scale of the participants not being a perfect asset for the platform.) This kind of admission control is what Apple is pursuing as a part of its Appstore policy. Selective permission for being a supplier to the platform (Appstore) alleviates the congestion effect and maintains a certain level of quality, which is essential for a healthy ecosystem.

\section{Conclusion}

This study presents a stylized model for dynamic nonlinear value creation across multiple players in a platform ecosystem. The proposed model incorporates two perspectives of firm theories to help understand the roles of a platform and the fundamental organizing principle of its ecosystem. RBV considers the participants-users and suppliers-as not only clients but also assets of the platform; meanwhile, TCV provides the reason for the existence of the platform: to reduce the transaction costs across the participants. Our models together with analyses and experiments suggest that the fundamental role of a platform lies in controlling the transactions across its participants, moving along the spectrum of market vs. hierarchy. Leveraging both market and organization, as platforms do, may provide a hint for a prototype of IT-intensive service operations encompassing newly emerging $\mathrm{O} 2 \mathrm{O}$ (online-to-offline) services.

Since our results are based on the stylized, conceptual model, they have some limits when applying to real cases. First, some simplifying assumptions in the model (e.g., Eq. (3)) may restrict platform's behavior and lead the entire ecosystem toward some specific directions. The context of this study is limited, too. For example, the current approach considers neither vertical differentiation strategy of the platform nor collaborations among the suppliers. Lastly, the current framework needs to be extended to incorporate competing platforms, which probably affects the entire dynamics.

\section{References}

[1] Allee, V. (2000). "Reconfiguring the value network," Journal of Business Strategy, 21(4), 36-39.

[2] Barney, J.B. (1986). "Strategic factor markets: Expectations, luck, and business strategy,” Mgmt. Science, 32(10), 1231-1241. [3] Barney, J.B. (1991). "Firm resources and sustained competitive advantage,” J. of Management, 17(1), 99-120.

[4] Boudreau, K. (2010). "Open platform strategies and innovation: Granting access vs. devolving control,” Mgmt. Science, 56(10), 1849-1872.

[5] Coase, R.H. (1937). "The nature of the firm,” Economica, 4(16), 386-405.

[6] Cusumano, M.A. (2010). "The evolution of platform thinking," Comm. of the ACM, 53(1), 32-34.

[7] Eisenmann, T. (2008). "Managing proprietary and shared platforms," California Management Review, 50(4), 31-53.

[8] Eisenmann, T., G. Parker, and M.W. van Alstyne (2006). "Strategies for two-sided markets," Harvard Business Review, Sept./Oct. Issue, 84(10), 92.

[9] El Sawy, O.A., A. Malhotra, Y-K. Park, and P.A. Pavlou (2010). "Seeking the configurations of digital ecodynamics: It takes three to tango,” Info. Sys. Research, 21(4), 835-848.

[10] Evans, D.S. and R. Schmalensee (2007). Catalyst Code: the Strategies behind the World's Most Dynamic Companies, Harvard Business School Press.

[11] Gawer, A. (Ed) (2009). Platform, Markets and Innovation, Edward Elgar.

[12] Gawer, A. and M.A. Cusumano (2008). "How companies become platform leaders?,” MIT Sloan Management Rev., Winter Issue, 28-35.

[13] Hagiu, A. (2009). "Proprietary vs. open two-sided platforms and social efficiency,” Harvard Business School, WP 09-113.

[14] Hagiu A. and J. Wright (2011). "Multi-sided platforms," Harvard Business School, WP 12-024.

[15] Iansiti, M. and R. Levien (2004). "Strategy as ecology,” Harvard Business Review, March/Apirl Issue, 68-78.

[16] Katz, M.L. and C. Shapiro (1985). "Network externalities, competition, and compatibility,” American Econ. Rev., 424-440.

[17] Kim, H., J-N. Lee, and J. Han (2010). "The role of IT in business ecosystems," Comm. of the ACM, 53(5), 151-156.

[18] Mars, M.M., J.L. Bronstein, and R.F. Lusch (2012). "The value of a metaphor: Organizations and ecosystems,” Organizational Dynamics, 41, 271-280.

[19] Moore, J.F. (2006). "Business ecosystems and the view from the firm,” Antitrust Bulletin, 51(1), 31-75.

[20] Moore, J.F. (1993). "Predators and prey: a new ecology of competition,” Harvard Business Review, 71(3), 75-83.

[21] Penrose, E. (1959). The Theory of the Growth of the Firm, Oxford University Press.

[22] Peppard, J. and A. Rylander (2006). "From value chain to value network: Insights for mobile operators," European Management Journal, 24, 128-141.

[23] Porter, M.E. (1980). Competitive Strategy: Techniques for Analyzing Industries and Competitors, Free Press.

[24] Rochet, J.C. and J. Tirole (2002). "Platform competition in twosided markets,” J. of Euro Economic Assoc., 1, 990-1029.

[25] Sun, M. and E. Tse (2009). "The resource-based view of competitive advantage in two-aided markets," J. of Management Studies, 46(1), 45-64.

[26] Teece, D., G. Pisano, and A. Shuen (1997). "Dynamic capabilities and strategic management," Strategic Management J., 18(7), 509-533.

[27] Tiwana, A., B. Konsynski, and A.A. Bush (2010). "Platform evolution: Coevolution of platform architecture, governance, and environmental dynamics,” Info. Sys. Research, 21(4), 675-687.

[28] Wernerfelt, B. (1984). "A resource-based view of the firm," Strategic Management J., 5, 171-180.

[29] Williamson, O.E. (1975). Markets and Hierarchies: Analysis and Antitrust Implications, The Free Press. 